\title{
Supervisory GPC and Evolutionary PI Controller for Web Transport Systems
}

\author{
N. Muthukumar ${ }^{1}$, Seshadhri Srinivasan $^{2}$, K. Ramkumar $^{1}$, P. \\ Kavitha $^{3}$, Valentina Emila Balas ${ }^{4}$
}

${ }^{1}$ Department of Electronics and Instrumentation Engineering, School of EEE, SASTRA University, Tirumalaisamudram, Thanjavur - 613 401, Tamilnadu, India, muthukumar.n@sastra.ac.in; ramkumar@eie.sastra.edu

${ }^{2}$ International Research Center, Kalasalingam University, Srivilliputtur, Anand Nagar, Krishnankoil, Chennai, Tamil Nadu 626126, India, and Universita Studi Degli Del Sannio, Engineering Department, Piazza Roma 21, Benevento, 82100, Italy,seshadhri.srinivasan@unisannio.it

\footnotetext{
${ }^{3}$ Robotics and Control Division, School of Electrical Engineering, Vellore Institute of Technology, Near Katpadi Rd, Vellore, Tamil Nadu 632014, India, kavitha.p@vit.ac.in

${ }^{4}$ Department of Automatics and Informatics, Aurel Vlaicu University of Arad, Bdul Revolutiei 77, 310130 Arad, Romania, balas@drbalas.ro
}

\footnotetext{
Abstract: Web Transport Systems (WTS) are used in material processing industries to maintain constant tension on the transported material (web), that is required for assuring material integrity and to reduce production down-time. Maintaining constant web tension in the presence of disturbance is a challenging task. This investigation presents a cascade control design, with online supervisory web tension control using Generalized Predictive Controller (GPC) in major loop and offline evolutionary optimization based PI controller in the inner loop. Two algorithms are used to tune the inner PI controller: Real coded Genetic Algorithm (RGA) and Bacterial Foraging Particle Swarm Optimization (BF-PSO), due to their ability to solve non-linear optimization problems and convergence to global optimum. Our results indicate that the GPC-BF-PSO cascaded control design shows better performance by regulating tension without violating physical constraints in the presence of process and external disturbances, when compared to GPC-RGA cascaded control design.
}

Keywords: Web Transport System (WTS); Multivariable system; Generalized Predictive Controller (GPC); real-coded genetic algorithm (RGA); Bacterial Foraying Particle Swarm Optimization (BF-PSO); constrained optimization; cascaded architecture 


\section{Introduction}

Material processing industries such as paper, iron, aluminum, polystyrene etc., require transporting materials as sheets (also called as web) over long distances through various sections during manufacturing and finishing. As the web is subjected to tension variations and disturbances in the various sections, the integrity of the transported material gets affected. Traditionally, web tension controllers (WTCs) are used in process industries to regulate the tension. Performance improvements in WTC can lead to significant economic and quality benefits, and therefore, optimizing their performance in the face of emerging disturbances becomes significant. Further, such performance improvements should happen without any major hardware updates and investments. However, realizing such a controller is a challenging task due to the multi-variable nature of the web transport system and, the tight coupling among control variables and constraints.

Design of WTC has received considerable attention of researchers in the past two decades owing their wide applications and economic benefits. The available results can be broadly classified into five categories, they are: (i) conventional controllers (see, [9], [12], [16], [17], [27], [30], [31] and [34]), (ii) robust controllers (for e.g., [5], [8], [13] and [17]) (iii) adaptive controllers ([1] and [23]), (iv) optimizing controllers [33]. A comparison of these controllers highlighting their advantages and limitations is shown in Table 1.

Widely used conventional controllers are PID (proportional-integral-derivative) and their varieties (such as PI). Though, PID controllers are simple and cheap [3], they are subjected to limitations such as performance degradation in the presence of noise, parametric variations and multi-variable interaction, and moreover they are not optimal [7]. To address the issues related to parametric variations in PID controllers, adaptive Controllers based on MRAC has been proposed in [1] though these controllers are more suited for dealing with parameter uncertainties, they require online estimation of the model parameters and are not optimal. Robust $\mathrm{H}$ $\infty$ controllers proposed in [8] can deal with model uncertainties and the disturbances without employing online estimation. However, need for accurate models and complex computations make their implementation in process industries difficult. In spite of these results in designing WTC, there are two limitations in these methods that need to be addressed - first, the performance (these methods are not optimal) and second, they require significant hardware upgrades. Objective of this investigation is to design WTC that optimizes the product quality and energy cost in the presence of disturbances without major hardware updates.

To reach our first objective of optimizing the quality and energy cost, the investigation proposes to use model predictive controller (MPC) that not only combines the advantages of the existing results in adaptive and robust controller such as using models, disturbance attenuation, and dealing with model 
uncertainties, but also overcomes their shortcomings such as the need for accurate models, online estimation of model parameters and being not optimal. Recently, Dynamic Matrix Controller (DMC) for WTS proposed in [33] uses the model of the process along with constraints, estimate of the disturbance and an optimization routine to compute the future control moves in receding horizon manner to enhance the product quality and reduce energy cost. But, step response based computation in DMC involves intensive computations for multivariable process. On the other hand, the Generalized Predictive Controller (GPC) uses explicit optimization techniques with reduced computations is more suitable for multivariable process. The advantages of the GPC is that it requires only an approximate model, inherently handles disturbances, constraints (operational and physical) and other complex phenomenon (such as multi-variable systems and their interactions), and is optimal [25].

Table 1

Summary of controllers employed for web tension control in WTS

\begin{tabular}{|c|c|c|}
\hline CONTROLLER & ADVANTAGES & LIMITATIONS \\
\hline $\begin{array}{c}\text { Conventional } \\
{[9][12]} \\
{[16][17][27][30][31][34]}\end{array}$ & Simple and cheap & $\begin{array}{ll}\text { (i). } & \begin{array}{l}\text { Performance } \\
\text { degradation with } \\
\text { disturbance }\end{array} \\
\text { (ii). Not optimal }\end{array}$ \\
\hline $\begin{array}{l}\text { Adaptive } \\
\text { [1] [23] }\end{array}$ & $\begin{array}{l}\text { Accommodates parameter } \\
\text { uncertainties and disturbances }\end{array}$ & $\begin{array}{l}\text { (i). Not optimal } \\
\text { (ii). Online adaptation is } \\
\text { difficult }\end{array}$ \\
\hline $\begin{array}{c}\text { Robust } \\
{[5][8][13][17]}\end{array}$ & $\begin{array}{l}\text { Handling disturbances and } \\
\text { model uncertainties }\end{array}$ & $\begin{array}{l}\text { (i). Requires accurate } \\
\text { model } \\
\text { (ii). Complex computations } \\
\text { (iii). Implementation } \\
\text { requires hardware } \\
\text { updates }\end{array}$ \\
\hline $\begin{array}{l}\text { Optimizing Controllers } \\
\text { [33] }\end{array}$ & $\begin{array}{l}\text { (i). Guaranteed optimality } \\
\text { (ii). Requires only } \\
\text { approximate model } \\
\text { (iii). Handles the disturbances, } \\
\text { constraints and other } \\
\text { complex phenomenon } \\
\text { such as non-linearity, } \\
\text { multi-variable interactions } \\
\text { inherently }\end{array}$ & $\begin{array}{l}\text { (i). } \begin{array}{l}\text { Requires the solution of } \\
\text { optimization problem }\end{array} \\
\text { (ii). Convergence of the } \\
\text { optimization problem }\end{array}$ \\
\hline
\end{tabular}




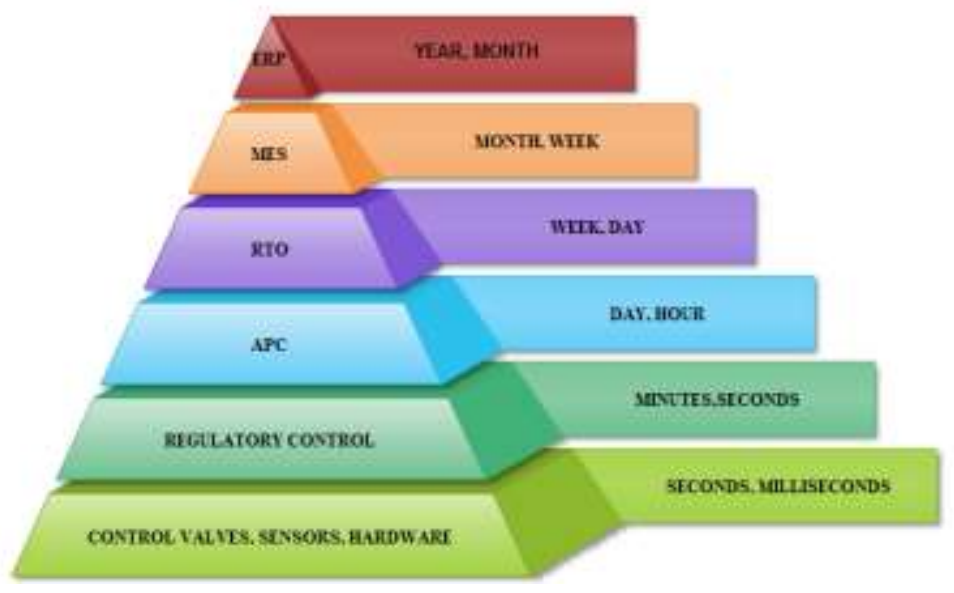

Figure 1

Hierarchical Control Architecture for mapping ISA 95 standard

In order to avoid hardware updates, a close look into the organization of the process industry is required. The organization of the process industry and the interfaces between the enterprise and control layer is defined by the standard ISA 95 [35]. The standard organizes the process industries into six layers that can be mapped to hierarchical control architecture shown in Figure 1. Within this architecture, the control between the supervisory and plant-floor is described the advanced process control (APC), regulatory, and field level control that work in different time-frames. Traditionally, MPCs are implemented in the APC layer due to the computation time required to solve the complex optimization problem, and to avoid costly hardware updates in the lower layers [36]. Therefore, this investigation proposes to use MPC in the APC layer, while the PI controller for performing the regulatory control is used for tension regulation. This leads to a cascaded architecture wherein the MPC is in the outer loop, while the PI controller is in the inner loop. The MPC working as a supervisory controller provides the references that need to be tracked by the regulatory PI controller. Here it should be stressed that, since the inner loop PI controller influences the performance of the cascaded controller, their parameters need to optimized. As the time-frames of regulatory control do not support on-line optimization, this investigation proposes to use off-line optimization. Though, there are many off-line tuning methods available in literature, evolutionary optimization have gained importance due to their ability to reach global solutions in reasonable time compared to conventional tuning methods [2]. Among the available EAs, Bacterial foraging Particle Swarm Optimization (BF-PSO) and Real coded Genetic Algorithms (GA) uses bacterial foraging technique and genetic operators, respectively to move towards global minimum with faster convergence rate. Hence, this investigation proposes to use RGA and BF-PSO for tuning the inner loop regulatory PID controller in the regulatory layer. 
The main contributions of this investigation are: (i) hierarchical control architecture following the ISA 95 for designing cascaded WTC, (ii) supervisory MPC controller for WTS that considers the operating and physical constraints, and (iii) Evolutionary Optimization based PI controller for regulating tension.

This paper is organized into six sections. Section 2 proposes the hierarchical layered controller design architecture, and Section 3 provides the mathematical model of the WTS. In Section 4, the implementation aspects of the cascaded controller are discussed, while Section 5 presents the results obtained with the proposed cascaded controller. Conclusions and future prospects of this investigation are discussed in Section 6.

\section{Hierarchical Controller Architecture for Web Transport System Design}

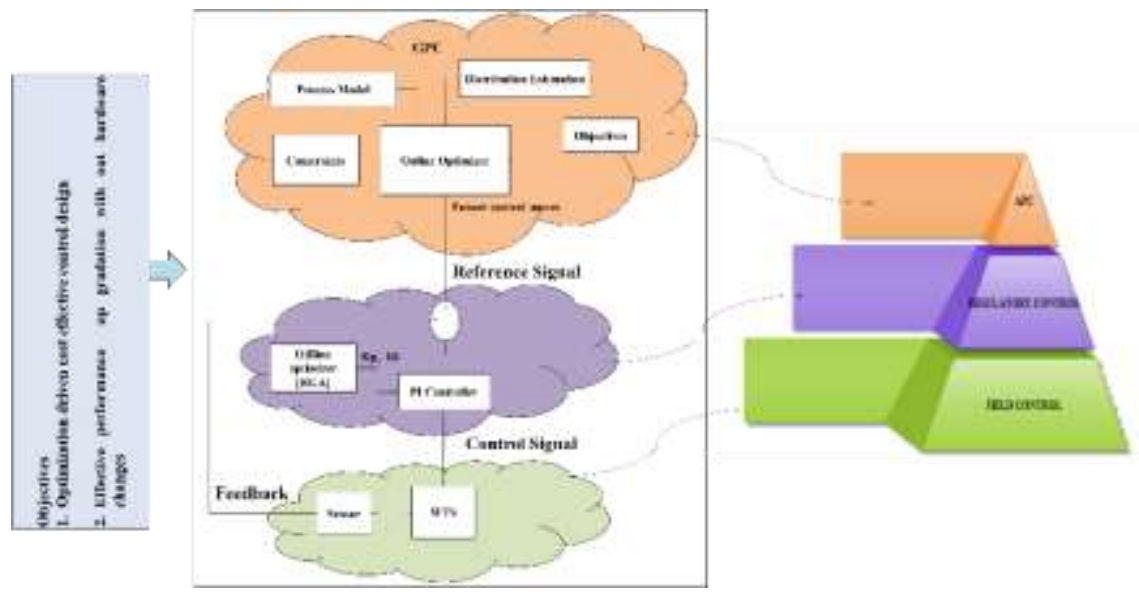

Figure 2

Cascaded Controller Architecture

The cascaded control architecture of the WTS that maps the hierarchical control in process industries to the objectives is shown in Figure 2. The APC, regulatory and field control layers work in different time-frames and the computation power of the APC layer is more than that of the regulatory layer. The APC layer consists of a GPC that works in a supervisory mode to maintain constant web tension by utilizing the model of the system, estimation on disturbances, information on constraints and an optimization routine to compute the future control inputs. The future control sequences are provided as the reference signals to the controller in regulatory layer. The regulatory control consists of offline optimized PI controller tuned by RGA and BF-PSO that uses the reference input from APC layer to control the velocity of the motors in the winder and un-winder to maintain the 
tension in the web. The field control layer uses the sensor (load-cell) to give the feedback to the regulatory layer that implements the closed-loop control. The advantage of using hierarchical architecture in WTS are twofold: (i) performance degradation due to parameter variations in WTS can be easily addressed using process model in APC layer by altering the reference signal provided to the regulatory layer, and (ii) effective disturbance rejection can be achieved by using hierarchical cascaded structure as the GPC in the APC layer can detect the disturbance early and change the reference input to the regulatory layer based on the predicted disturbance. To design the hierarchical controller, the mathematical model of WTS is necessary and the next section presents the mathematical model required for designing the GPC.

\section{Mathematical Model of Web Transport System}

This section describes the web transport system and derives the mathematical model required for designing the MPC. The variables and constants of the model along with their respective units is given in Table 2 .

Table 2

List of Symbols used in mathematical model of WTS

\begin{tabular}{|c|c|}
\hline SYMBOLS & DESCRIPTION \\
\hline $\mathrm{L}$ & Length of the web in $\mathrm{m}$ \\
\hline $\mathrm{T}$ & Tension in the web in $\mathrm{N}$ \\
\hline $\mathrm{E}$ & Young's modulus in $\mathrm{N} / \mathrm{m}$ \\
\hline $\mathrm{A}$ & Cross sectional area of the web in $\mathrm{m}^{2}$ \\
\hline $\mathrm{V}_{1}$ & Velocity of the un-winder roller in $\mathrm{m} / \mathrm{s}$ \\
\hline $\mathrm{V}_{2}$ & Velocity of the winder roller in $\mathrm{m} / \mathrm{s}$ \\
\hline$\tilde{T}$ & Time derivative of the tension of the web in $\mathrm{N} / \mathrm{s}$ \\
\hline $\mathrm{R}$ & Radius of the roller in $\mathrm{m}$ \\
\hline $\mathrm{N}_{\mathrm{g}}$ & Moment of inertial of the motor in $\mathrm{Nm}{ }^{2}$ \\
\hline$l_{m}$ & Viscous friction constant of the motor in $\mathrm{Nm} . \mathrm{s} / \mathrm{rad}$ \\
\hline$B_{m}$ & Torque of the motor in Nm \\
\hline$\tau_{m}$ & Angular Velocity of the motor in rad $/ \mathrm{s}$ \\
\hline$\omega_{m}$ & Time derivative of velocity of the motor in rad $/ \mathrm{s}^{2}$ \\
\hline$\omega_{m}$ & Steady state velocity of the winder motor in $\mathrm{m} / \mathrm{s}$ \\
\hline$V_{2 s s}$ & Steady state velocity of the un-winder motor in $\mathrm{m} / \mathrm{s}$ \\
\hline$V_{1 s s}$ &
\end{tabular}


The WTS consists of a winder and un-winder section coupled through the moving web and passing via the pinch rollers. As the effect of pinch rollers on the moving web is found to be negligible from our analysis, it is therefore not included in our model as shown in Figure 3. The web tension is maintained by adjusting the roller velocity that is coupled to the electric motor using gears. This arrangement of the WTS leads to a multi-variable system having winder and un-winder velocities as inputs, while the tension measured using a load cell in winder and un-winder section are the outputs.

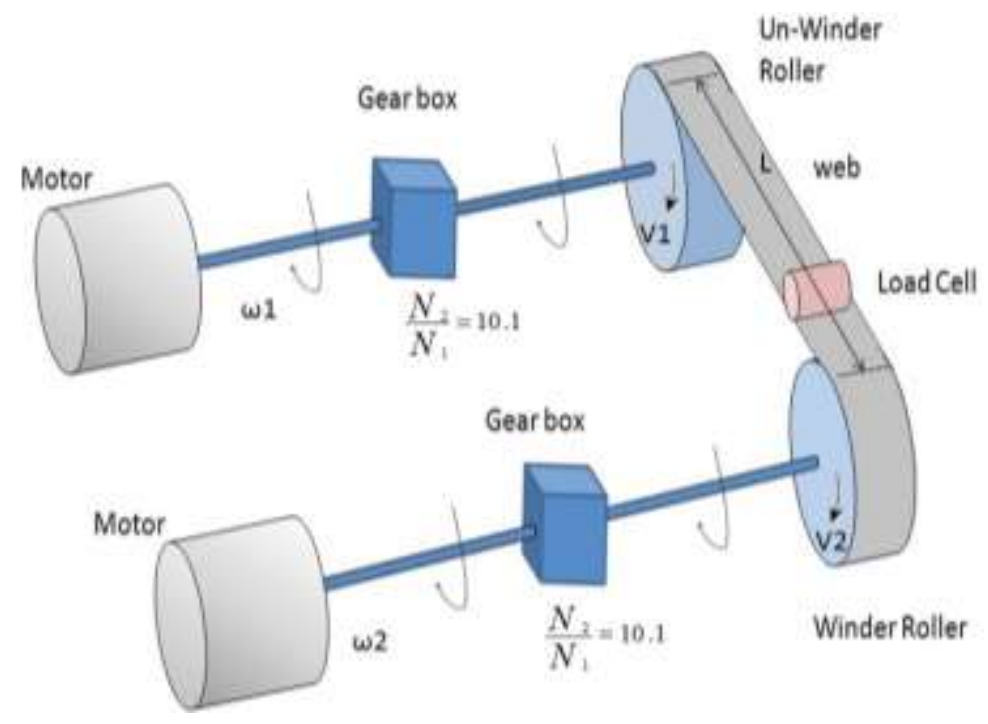

Figure 3

A Schematic of a rudimentary Web Transport System

The multivariable mathematical model of WTS indicated in Figure 4 is derived from law of conservation of mass and Hooks law [19]. The dynamics of the web material is given by [8]

$\stackrel{\hat{T}}{=}=-\frac{\mathbb{V}_{2 n s}}{L} \hat{T}+\frac{A E}{L}\left[\hat{V}_{2}-\hat{V}_{1}\right]$

where, $\hat{T}=t-t_{g s}, \hat{V}_{2}=V_{2}-V_{2 s g}$, and $\hat{V}_{1}=V_{1}-V_{1 s g}$, represents the linearized tension and velocity variables.

The mathematical relations between angular velocity and torque of the motor (2), the angular velocity of the motor and the tangential velocity of the roller (3) given by [6]

$\omega_{m}=-\frac{B_{m}}{h_{m}} \omega_{m}+\frac{1}{h_{m}} \tau_{m}$

$V_{2}=\frac{R}{N_{g}} \omega_{m}$ 
The state space model describing the WTS can be derived from equations (1), (2) and (3) as:

$$
\left.\begin{array}{c}
\stackrel{x}{T}=\left[\frac{-V_{2 x}}{L}\right] \widehat{T}+\left[\begin{array}{ll}
\frac{-A E}{L} & \frac{A E}{L}
\end{array}\right]\left[\begin{array}{l}
V_{1} \\
V_{2}
\end{array}\right] \\
y=\hat{T}
\end{array}\right\}
$$

where, $\mathrm{V}_{1}$ and $\mathrm{V}_{2}$ are the control inputs and $\widehat{T}$ is the output.

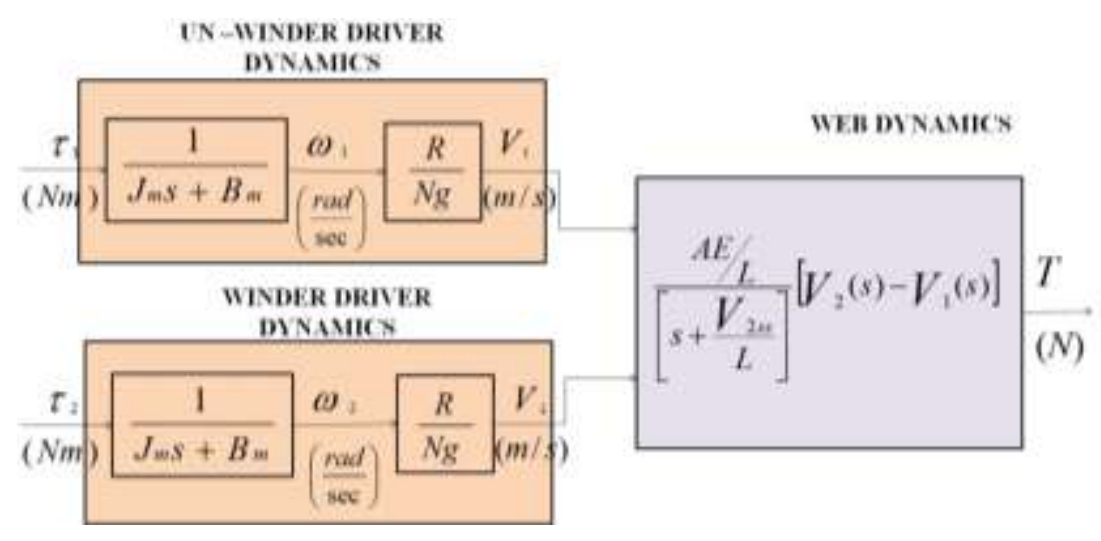

Figure 4

Block diagram representation of WTS with multivariable interactions

\section{Cascaded Control Design for WTS}

Having obtained the mathematical model of WTS, the design of hierarchical supervisory controller is discussed in this section. This section presents the cascaded control design that uses the GPC in the outer loop working in a supervisory mode, and evolutionary optimized PI controller in the inner loop that operates as a regulatory controller, as shown in Figure 5. In order to tune the inner loop PI controller, many methods exists in literature. However, optimization based tuning are used to improve the performance. Although many optimization based tuning methods exists, evolutionary optimization based tuning have evolved as promising solutions due to their ability to handle non-linear objective functions, and reaching global optimization. Two most common evolutionary computing algorithms used for tuning PID controllers are the genetic operators based RGA and BF-PSO. The off-line optimizer in this investigation is equipped to tune the inner loop based on these algorithms. The performance of these two algorithms can be compared and used appropriately. The inner loop controllers adjust the roller speeds by taking the reference inputs from the GPC. The control design for supervisory controller and regulatory controller is explained in the subsection 4.1 and 4.2 respectively. 


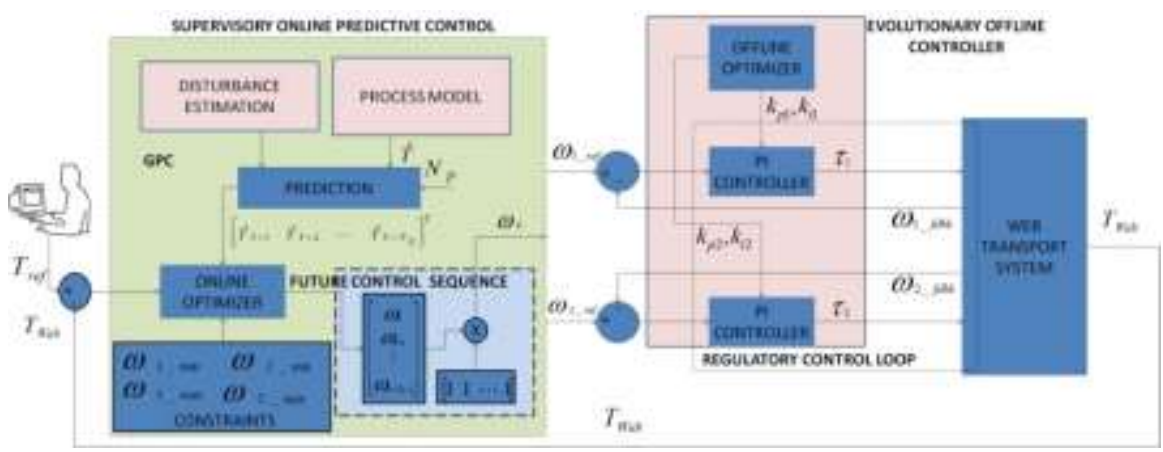

Figure 5

Functional block diagram of cascade control in a Web Transport System

\subsection{Online Supervisory Predictive Control}

The roller velocity in WTS plays a critical role to ensure constant web tension. Any deviation from the normal operation in roller velocity will affect the integrity and quality of the web significantly. Moreover, the WTS is subjected to various disturbances that lead to web degradation. Hence, an online optimization based control strategy that ensures constant web tension and disturbance rejection with minimum control effort, considering physical constraints needs to be employed. In this backdrop, the GPC algorithm that uses optimization techniques and process models to predict the future response to compute control input can be employed in the APC layer as a supervisory control to regulate roller velocities of WTS. The GPC implementation depends on the prediction matrices that can be computed off-line (explicit GPC), and this reduces computations significantly.

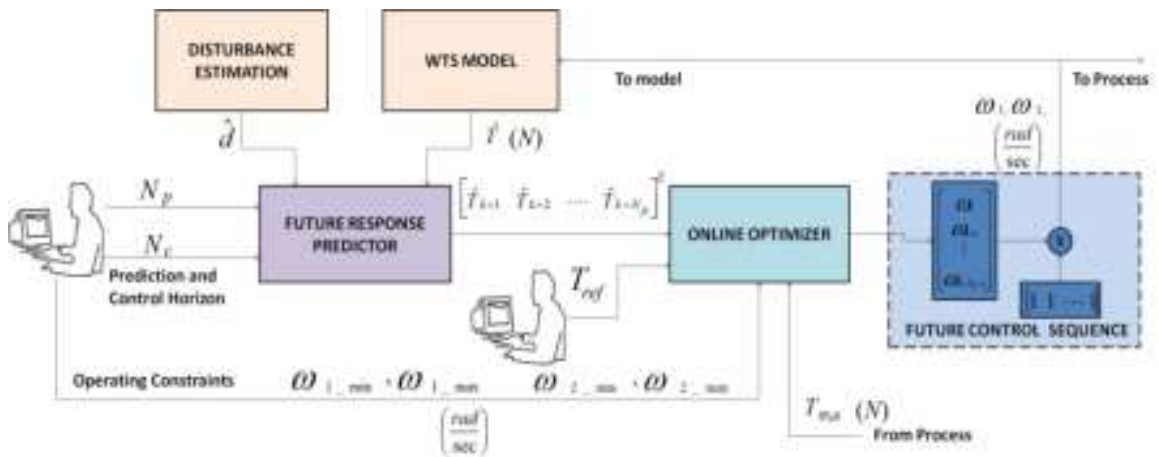

Figure 6

Block diagram representation of GPC algorithm

The GPC algorithm was proposed by Clarke. et.al [6] has many industrial applications [10] [19]. In GPC, the future response of a process for a time line $\mathrm{Np}$ 
(prediction horizon) can be predicted with the help of the process model (see [18] and [25] for detailed derivation). The state space model of the WTS is given by

$$
\left.\begin{array}{c}
x_{k}=\left[\begin{array}{c}
T(k+1) \\
u(k)
\end{array}\right]=\left[\begin{array}{cc}
A & B \\
0 & I
\end{array}\right]\left[\begin{array}{c}
T(k) \\
u(k-1)
\end{array}\right]+\left[\begin{array}{l}
B \\
I
\end{array}\right] \Delta u(k) \\
y(k)=\left[\begin{array}{ll}
C & D
\end{array}\right]\left[\begin{array}{c}
T(k) \\
u(k-1)
\end{array}\right]+D \Delta u(k)
\end{array}\right\}
$$

Here,

$$
\begin{aligned}
& \hat{A}=\left[\begin{array}{ll}
A & B \\
0 & I
\end{array}\right] ; \quad \widehat{B}=\left[\begin{array}{l}
B \\
I
\end{array}\right] ; \quad \hat{C}=\left[\begin{array}{ll}
C & D
\end{array}\right] \\
& O^{T}=\left[\begin{array}{lll}
0 & 0 & \ldots
\end{array}\right]
\end{aligned}
$$

The prediction model to determine future response for (5) is given as

$$
Y=F \hat{T}(k)+\Phi \Delta U
$$

where

$$
\begin{aligned}
& Y=\left[y(k+1 \mid k) y(k+2 \mid k) \ldots y\left(k+N_{p} \mid k\right)\right]^{T} \\
& \hat{T}(k)=\left[\begin{array}{llll}
T(k+1 \mid k) & T(k+2 \mid k) & \text {. } & T\left(k+N_{p} \mid k\right)
\end{array}\right]^{T} \\
& \Delta U=\left[\Delta u(k) \Delta u(k+1) \Delta u(k+2) \ldots \Delta u\left(k+N_{p}+1\right)\right]^{T} \\
& F=\left[\begin{array}{c}
\hat{C} \hat{A} \\
\hat{C} \hat{A}^{2} \\
\hat{C} \hat{A}^{a} \\
\vdots \\
\hat{C} \hat{A}^{N_{F}}
\end{array}\right] ; \Phi=\left[\begin{array}{ccccc}
\hat{C} \hat{B} & 0 & 0 & \ldots & 0 \\
\hat{C} \hat{A} \hat{B} & \hat{C} \hat{B} & 0 & \ldots & 0 \\
\hat{C} \hat{A}^{2} \hat{B} & \hat{C} \hat{A} \hat{B} & \hat{C} \hat{B} & \ldots & 0 \\
\vdots & \vdots & \vdots & \vdots & \vdots \\
\hat{C} \hat{A}^{N_{F}-1} \hat{B} & \hat{C} \hat{A}^{N_{F}-2} \hat{B} & \hat{C} \hat{A}^{N_{P}-1} \hat{B} & \ldots & \hat{C} \hat{A}^{N_{P}-N_{C} \hat{B}}
\end{array}\right]
\end{aligned}
$$

Here, $\phi$ is a positive definite matrix and it is called prediction matrix. $\mathrm{F}$ is positive semi definite matrix.

The quadratic objective function $\mathrm{J}$ is formulated with the predicted model, process output and control input. The objective function is solved online for an optimum value of $\Delta U$ subject to the operating constraints as given below

$$
\min _{\Delta U} J=\Delta U^{T} H \Delta U+f^{T} \Delta U \quad \text { s.t } G \Delta U-L_{k} \leq 0
$$

where, $H=\left(\Phi^{\mathrm{T}} \Phi+\lambda \mathrm{I}\right) ; \quad f^{T}=2 \Phi^{\mathrm{T}}\left(R_{g}-F x_{k}\right) ; \quad R_{S}{ }^{T}=[111 \ldots 1] r(k)$ $\lambda$ is scaling factor of the input. The operating constraints are represented as

$$
G=\left[\begin{array}{c}
I \\
-I \\
G_{I / \Delta} \\
-G_{I / \Delta} \\
\Phi \\
-\Phi
\end{array}\right] ; L_{k}=\left[\begin{array}{c}
\Delta U_{\max } \\
-\Delta U_{\min } \\
u_{\max } \\
-u_{\min } \\
y_{\max } \\
-y_{\min }
\end{array}\right]
$$


where, $r(k)$ is the reference value, $I$ is the identity matrix, $G_{I / \Delta}$ is the lower triangular matrix, $\Delta U_{\max }$ and $\Delta U_{\min }$ are the limits for change in control input; $u_{\max }$ and $u_{\min }$ are the input limits; $y_{\max }$ and $y_{\min }$ are the output limits.

The objective function yields optimal future control inputs for the next $\mathrm{Np}$ steps. The first among the control moves computed is applied to the process and the rest is discarded. The process is repeated in receding horizon manner.

\subsection{Offline Optimized Regulatory Controller}

The use of evolutionary optimization to design PID controllers for the inner loop regulatory control is explored in this section. First, the working of genetic operator based RGA is presented, and then BF-PSO algorithm is discussed in detail. The off-line optimizer block in Figure 5, is equipped with the two evolutionary algorithms and can be used to tune the inner loop controller.

\subsubsection{Regulatory Controller Design using RGA}

The RGA uses real values as against the use of binary values in traditional GA. This makes computations simple due to the absence of conversion and reconversion. RGA has been used in many applications (see [9], [20], [24], [21] and [29] for details). RGA employs biological methods such as crossover, mutation and reproduction to produce globally optimized PI controller parameters (kp and ki) by simultaneously minimizing a fitness function. In our investigation, IAE is chosen as the fitness function (F) due to its capability to penalize errors in both transient and steady state response. The expression of IAE to penalize errors occurring due to roller velocity deviation from reference velocity is given as

$F=\sum_{i=1}^{2} a b s\left(\omega_{i_{-} r e f}-\omega_{i}\right)$

where, $\omega_{\mathrm{i}}$ ref is the reference roller velocity and $\omega_{\mathrm{i}}$ is the feedback roller velocity from the process; $i=1,2$ represents the un-winder and the winder rollers respectively.

In RGA, initially a random population is created (also call the parent) of the controller parameters are generated. Then, using the parent samples, the fitness function is evaluated and ranked. The best species are selected and the ones that lack optimality are discarded. The discarded population is replaced by new sample points and the old parent population is replaced with the new generation. Then genetic operators, mutation and cross-over are applied. This procedure continues until a reasonably accurate (specified by the user) solution is found. The RGA can acheive global minimum due to the presence of genetic operators that create new generation that are quite different from the parent population. 


\subsubsection{Regulatory Control Using BF-PSO}

The BF-PSO algorithm combines the foraging technique used by the E coli bacteria to minimize the cost function (8) and move towards the global optimization (see [2], [15], [24] and [20] for details). The bacterial foraging includes swimming, tumbling, reproduction, elimination and dispersal [3]. Initially a sample bacteria population (s) is chosen and chemotaxis $\left(\mathrm{N}_{\mathrm{c}}\right)$ step is carried out. The Chemotaxis $\left(\mathrm{N}_{\mathrm{c}}\right)$ steps involve swimming and tumbling process $\left(\mathrm{N}_{\mathrm{s}}\right)$. In a search space, if more feasible solutions are available, then the swimming process is enabled so as to move the population towards the global optimization. On the other hand, if there are no feasible solutions, the search direction of the population is tweaked by a small amount termed as tumble. The cost function (8) is solved and the position and the velocity (search direction) of the bacteria are calculated.

After a predetermined chemotaxis $\left(\mathrm{N}_{\mathrm{c}}\right)$ step $\left(\mathrm{N}_{\mathrm{s}}\right)$, the bacterial populations are ranked based on the their health. The population (s) with high health value is retained are allowed to reproduce and the reproduced population $\left(S_{\mathrm{r}}\right)$ search towards the global optimization. The elimination and dispersion process $\left(\mathrm{N}_{\mathrm{ed}}\right)$ are used to kill or disperse the population in a search area to end the chemo taxis process. The process of dispersion places the population close to the global optimization in a search space. This process is carried out for a fixed number of iterations to reach the global optimized PI controller parameters.

\section{Results and Discussion}

To illustrate the working of the cascade controllers, two scenarios are considered: GPC working as supervisory controller- and inner PI controller tuned using RGA and the one tuned using BF-PSO.

Table 3

Parameters of WTS used in simulation

\begin{tabular}{|c|c|}
\hline PARAMETER & VALUE \\
\hline $\mathrm{E}$ & $4 \times 10^{9} \mathrm{~N} / \mathrm{m}^{2}$ \\
\hline$I_{\mathrm{m}}$ & $3.1 \times 10^{-3} \mathrm{Kg} \cdot \mathrm{m}^{2}$ \\
\hline$B_{\mathrm{m}}$ & $0.55 \times 10^{-3} \mathrm{Nm} . \mathrm{s} / \mathrm{rad}$ \\
\hline $\mathrm{L}$ & $0.61 \mathrm{~m}$ \\
\hline $\mathrm{A}$ & $4.35 \times 10^{-6} \mathrm{~m}^{2}$ \\
\hline $\mathrm{R}$ & $57.3 \times 10^{-3} \mathrm{~m}$ \\
\hline $\mathrm{N}_{\mathrm{g}}$ & 10.1 \\
\hline$V_{2 g s}$ & $0.2 \mathrm{~m} / \mathrm{s}$ \\
\hline
\end{tabular}


Table 4

GPC control parameters for web tension control in WTS

\begin{tabular}{|c|c|}
\hline PARAMETER & VALUE \\
\hline Prediction Horizon & 5 \\
\hline Control Horizon & 2 \\
\hline$\lambda$ & 0.03 \\
\hline
\end{tabular}

Table 5

Constraints imposed on GPC for web tension control in WTS

\begin{tabular}{|c|c|}
\hline CONSTRAINTS & VALUE \\
\hline $\mathrm{V} 1_{\max }$ & $0.1 \mathrm{~m} / \mathrm{sec}$ \\
\hline $\mathrm{V} 1_{\min }$ & $0 \mathrm{~m} / \mathrm{sec}$ \\
\hline $\mathrm{V} 2_{\max }$ & $0.2 \mathrm{~m} / \mathrm{sec}$ \\
\hline $\mathrm{V} 2_{\min }$ & $0 \mathrm{~m} / \mathrm{sec}$ \\
\hline
\end{tabular}

The step response for reference web tension of $90 \mathrm{~N}$ using cascaded GPC- PI tuned using RGA and cascaded GPC - PI tuned using BF-PSO is shown in Figure 7. The GPC-BF-PSO cascaded controller exhibits better transient characteristics than GPC- RGA cascaded one. Comparison of rise-time, peak-overshoot, and settling time of the cascaded controller with inner loop RGA based PI and BFPSO based PI controller is shown in Table 6. Further, BF-PSO shows significant improvements in performance metrics such as IAE, ITAE, ISE, and ITSE as against RGA based PI as indicated in Figures 9 and 10, respectively.

As for, disturbance rejection, a step disturbance of magnitude $0.001 \mathrm{~m} / \mathrm{s}$ is provided to un-winder roller velocity at time $t=40$. It is found that the GPC $-\mathrm{BF}$ PSO provides better disturbance rejection compared to GPC-RGA architecture as indicated in Figure 8.

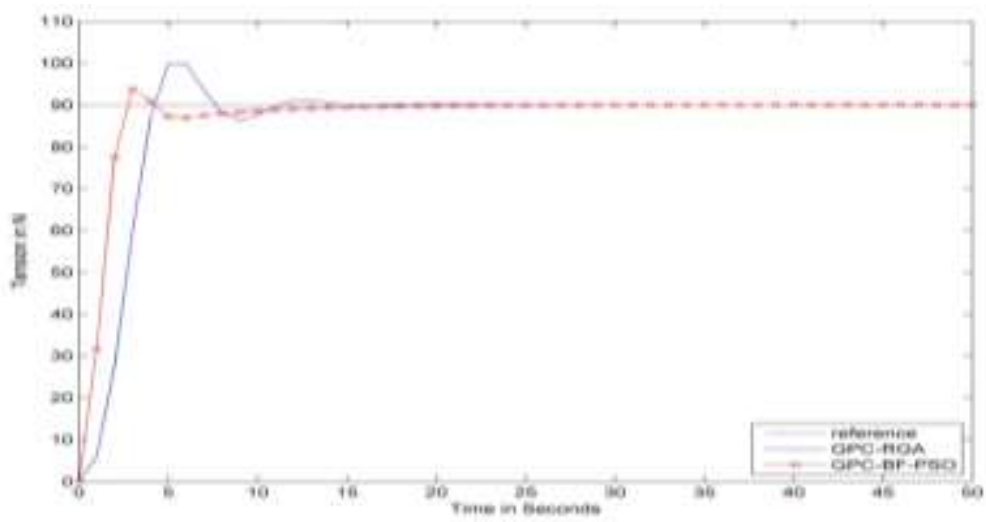

Figure 7

Responses of GPC - RGA architecture and GPC -BF-PSO for a reference tension of $90 \mathrm{~N}$ 


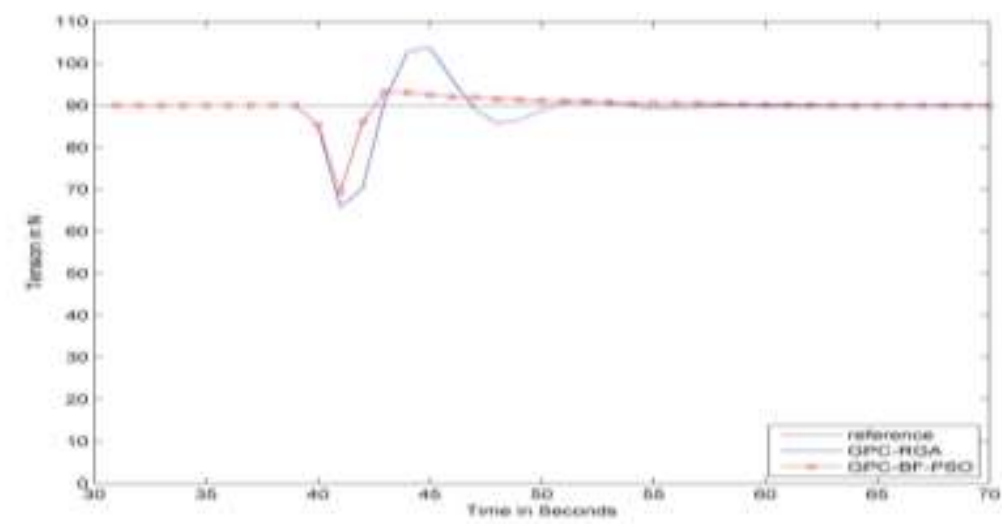

Figure 8

Web Tension disturbance rejection for a step disturbance of $0.0001 \mathrm{~m} / \mathrm{s}$ at time $\mathrm{t}=40 \mathrm{sec}$ in un-winder roller velocity using GPC-RGA and GPC-BF-PSO architecture

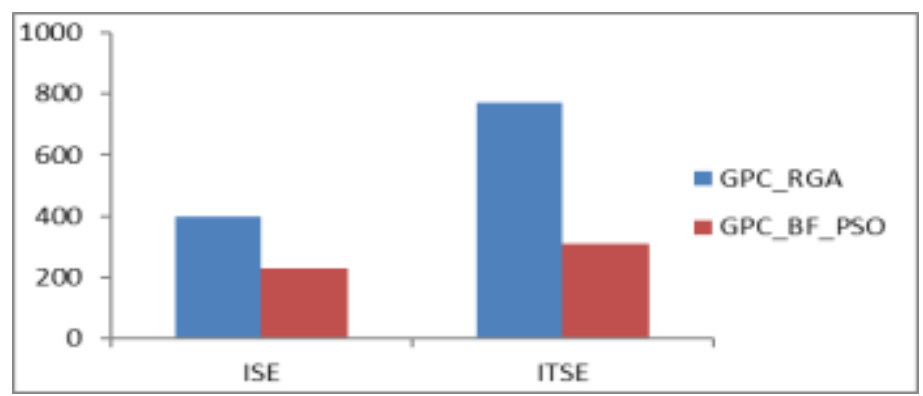

Figure 9

Comparison of Performance indices ISE and ITSE for GPC - RGA and GPC-BF-PSO architectures for a step change of $90 \mathrm{~N}$

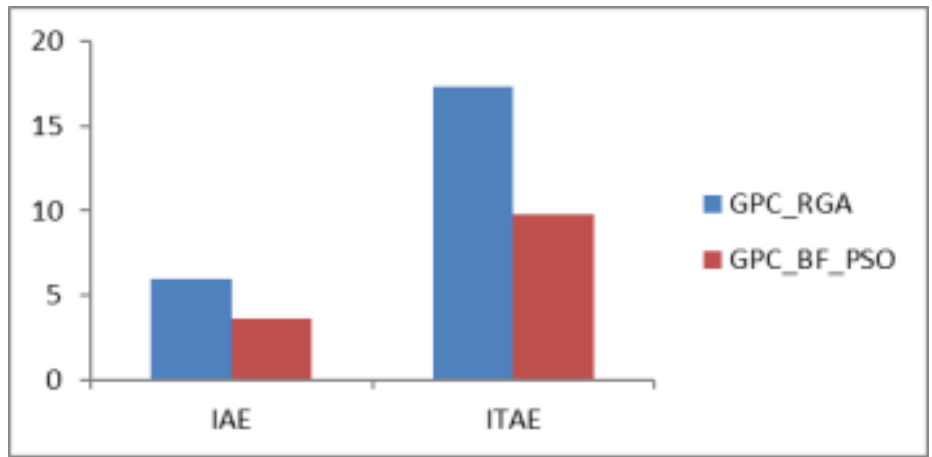

Figure 10

Comparison of Performance indices IAE and ITAE for GPC - RGA and GPC-BF-PSO architectures for a step change of $90 \mathrm{~N}$ 
Table 6

Comparison of transient performances obtained from GPC - RGA and GPC - BF-PSO control architectures for a step reference of $90 \mathrm{~N}$

\begin{tabular}{|c|c|c|c|}
\hline CONTROLLER & $\begin{array}{c}\text { RISE TIME } \\
\text { IN SECONDS }\end{array}$ & $\begin{array}{c}\text { SETTLING } \\
\text { TIME IN } \\
\text { SECONDS }\end{array}$ & $\begin{array}{c}\text { PEAK } \\
\text { OVERSHOOT } \\
\text { IN } \%\end{array}$ \\
\hline GPC - RGA & 3.7 & 35 & 11 \\
\hline GPC BF-PSO & 3.2 & 37 & 4 \\
\hline
\end{tabular}

Table 7

Comparison of performance indices obtained from GPC - RGA and GPC - BF-PSO control architectures for a step reference $90 \mathrm{~N}$

\begin{tabular}{|c|c|c|c|c|}
\hline CONTROLLER & IAE & ITAE & ISE & ITSE \\
\hline GPC - RGA & 5.99 & 17.32 & 396.72 & 770.89 \\
\hline GPC BF-PSO & 3.65 & 9.78 & 229.91 & 309.84 \\
\hline
\end{tabular}

The improvement in the performance of GPC -BF- PSO architecture over GPC RGA architecture is primarily due to the effectiveness of the regulatory control tuning using BF-PSO rather than RGA. The BF- PSO provides better minimization of cost function as indicated in Table 8 and converges towards global optimum with a small population size and minimum iterations. On the other hand, the RGA uses a population size that is $90 \%$ higher than BF-PSO and requires more iteration that amounts to $80 \%$ increase as against BF -PSO. The PI controller gains with RGA and BF-PSO algorithm are shown in Table 9.

Table 8

RGA and BF-PSO performance and parameters

\begin{tabular}{|c|c|c|c|}
\hline ALGORITHM & COST FUNCTION & POPULATION SIZE & $\begin{array}{c}\text { NO. OF } \\
\text { ITERATIONS }\end{array}$ \\
\hline RGA & 0.1215 & 20 & 20 \\
\hline BF-PSO & 0.1126 & 2 & 4 \\
\hline
\end{tabular}

Table 9

PI controller gains obtained using RGA and BF-PSO algorithm

\begin{tabular}{|c|c|c|}
\hline $\begin{array}{c}\text { CONTROLLER } \\
\text { ALGORITHM }\end{array}$ & UNWINDER & WINDER \\
\hline \multirow{2}{*}{ RGA } & $\mathrm{k}_{\mathrm{p}}=0.0051$ & $\mathrm{k}_{\mathrm{p}}=0.0130$ \\
\cline { 2 - 3 } & $\mathrm{k}_{\mathrm{i}}=0.0099$ & $\mathrm{k}_{\mathrm{i}}=0.0098$ \\
\hline \multirow{2}{*}{ BF-PSO } & $\mathrm{k}_{\mathrm{p}}=0.0059$ & $\mathrm{k}_{\mathrm{p}}=0.0356$ \\
\cline { 2 - 3 } & $\mathrm{k}_{\mathrm{i}}=0.0038$ & $\mathrm{k}_{\mathrm{i}}=0.0034$ \\
\hline
\end{tabular}




\section{Conclusion}

This investigation presented a cascaded controller for WTS based on ISA 95 architecture that effectively performs disturbance rejection, and good tension regulation without significant hardware upgrades. The cascaded controller uses supervisory GPC controller and off-line optimized PI controller. The GPC controller uses the model, disturbance estimation, knowledge of constraints and an optimization routine to reduce the energy consumed to maintain tension. While, the inner loop PI controller works as a regulatory controller. The controller is tuned using off-line evolutionary optimization, due to the ability of EAs to handle non-linear objectives and computation simplicity in reaching global optimum. The performance of the proposed cascaded controller with the GPC in the outer loop and the inner loop tuned using RGA and BF-PSO is compared. Our results indicate that the BF-PSO performs better than RGA tuned PI, and the performance improvements from 39-60\% can be achieved with the cascaded architecture. Verifying the cascaded controller in an industry is the future course of this investigation.

\section{Acknowledgement}

The authors would like to express their gratitude to SASTRA UNIVERSITY for their support and motivation throughout this investigation.

\section{References}

[1] Baosheng Wang, Jianmin Zuo, Mulan Wang, Hongyan Hao: Model Reference Adaptive Tension Control of Web Packaging Material, Intelligent Computation Technology and Automation (ICICTA), 2008 International Conference on, Vol. 1, No., pp. 395-398, 20-22 Oct. 2008

[2] Biswas, A., Dasgupta, S., Das, S., Abraham, A.: Synergy of PSO and Bacterial Foraging Optimization-A Comparative Study on Numerical Benchmarks, Innovations in Hybrid Intelligent Systems, Springer Berlin Heidelberg, pp. 255-263, 2007

[3] Chandra Sekhar Mohanty; Partha Sarathi Khuntia; D. Mitra: A Modified Bacterial Foraging Optimised PID Controller for Time Delay Systems, International Journal of Advanced Intelligence Paradigms (IJAIP), Vol. 6, No. 4, 2014

[4] Chopra, Vikram, Sunil K. Singla, and Lillie Dewan: Comparative Analysis of Tuning a PID Controller using Intelligent Methods, Acta Polytechnica Hungarica, 11.8, 235-248, 2014

[5] Claveau, F., Chevrel, P., Knittel, D.: A Two Degrees of Freedom $\mathrm{H}_{2}$ Controller Design Methodology for Multi-Motors Web Handling System, Proceedings of the 2005 American Control Conference, pp. 1383-1388, Vol. 2, 8-10 June 2005 
[6] D. W.Clarke, C. Mohtadi and P. S. Tuffs: Generalized Predictive ControlPart I .The Basic Algorithm, Automatica, Vol. 23, No. 2, pp. 137-148, 1987

[7] Garelli, R. J, Mantz, H, De Battista: Limiting Interactions in Decentralized Control of MIMO Systems, Journal of Process Control, Vol. 16, pp. 473483, 2006

[8] Gassmann, V., Knittel, D, Pagilla, P. \& Bueno, M.: A Fixed-Order H_o Tension Control in the Unwinding Section of a Web Handling System Using a Pendulum Dancer, IEEE Transactions on Control Systems Technology, 20, 173-180, 2012

[9] Hou, Y., Gao, Z., Jiang, F., Boulter, B. T: Active Disturbance Rejection Control for Web Tension Regulation. Proceedings of the $40^{\text {th }}$ IEEE Conference on Decision and Control, Vol. 5, IEEE, 2001

[10] Jakel, Milan, and Petr Sosík: Genetically Evolved Agents for Stock Price Prediction, Acta Polytechnica Hungarica, 10.2, 21-35, 2013

[11] S. Joe Qina, Thomas A. Badgwell,: A Survey of Industrial Model Predictive Control Technology, Control Engineering Practice, Vol. 11, pp. 733-764, 2003

[12] Kim, Jeetae: Development of Hardware Simulator and Controller for Web Transport Process. Journal of Manufacturing Science and Engineering, 128.1, 378-381, 2006

[13] Knittel, D., Laroche, E., Gigan, D. \& Koc, H.: Tension Control for Winding Systems with Two-Degrees-of-Freedom $\mathrm{H} \propto$ Controllers, IEEE Transactions on Industry Applications, 39, 113-120, 2003

[14] Koc H.; Knittel D., de Mathelin M. \& Abba, G.: Modeling and Robust Control of Winding Systems for Elastic Webs, IEEE Transactions on Control Systems Technology, 10, 197-208, 2002

[15] Korani, Wael Mansour, Hassan T. Dorrah, and Hassan M. Emara: Bacterial Foraging Oriented by Particle Swarm Optimization Strategy for PID Tuning. International Symposium on Computational Intelligence in Robotics and Automation (CIRA) 2009 IEEE

[16] Ku Chin Lin: Frequency-Domain Design of Tension Observers and Feedback Controllers with Compensation, IEEE IECON 2002 [28 $8^{\text {th }}$ Annual Conference of the Industrial Electronics Society], Vol. 2, No., pp. 16001605, 5-8, Nov. 2002

[17] Ku Chin Lin: Observer-based Tension Feedback Control with Friction and Inertia Compensation, IEEE Transactions on Control Systems Technology, Vol. 11, No. 1, pp. 109-118, Jan 2003

[18] Liuping Wang: Model Predictive Control System Design and Implementation using Matlab, Springer, 2009 
[19] Mark L. Darby, Michael Nikolaou: MPC: Current Practice and Challenges, Control Engineering Practice, Vol. 20, pp. 328-342, 2012

[20] Mellal, Mohamed Arezki: Optimal Policy for the Replacement of Industrial Systems Subject to Technological Obsolescence-Using Genetic Algorithm, Acta Polytechnica Hungarica, 10.1: 197-208, 2013

[21] Mirko Ficko, Joze Balic, Miran Brezocnik, Ivo Pahole: Solving of Floor Layout Problem in Flexible Manufacturing System by Genetic Algorithms, International Journal of Advanced Intelligence Paradigms (IJAIP), Vol. 2, No. 4, 2010

[22] Mohanty, Chandra Sekhar, Partha Sarathi Khuntia, and D. Mitra: A Modified Bacterial Foraging Optimised PID Controller for Time Delay Systems, International Journal of Advanced Intelligence Paradigms, 6.4: 255-271, 2014

[23] Pagilla P., Dwivedula R. \& Siraskar, N.: A Decentralized Model Reference Adaptive Controller for Large-Scale Systems, IEEE/ASME Transactions on Mechatronics, 12, 154-163, 2007

[24] Passino, K. M.: Biomimicry of Bacterial Foraging for Distributed Optimization and Control, IEEE Control Systems Magazine, 52-67, 2002

[25] Riesco, Felix: Genetic Algorithm as Discrete-Time Filter Solver, International Journal of Advanced Intelligence Paradigms, 1, 3, 275-290, 2009

[26] J. A. Rossiter: Model-based Predictive Control - A Practical Approach, CRC press, 2004

[27] Sakamoto, Tetsuzo, and Yoshikazu Fujino: Modelling and Analysis of a Web Tension Control System, Proceedings of the IEEE International Symposium on Industrial Electronics, IEEE ISIE'95, Vol. 1, 1995

[28] Sakamoto T. \& Izunihara Y.: Decentralized Control Strategies for Web Tension Control System, Proceedings of the IEEE International Symposium on Industrial Electronics, ISIE '97, 1086-1089, Vol. 3, 1997

[29] Slivka, Jelena, Aleksandar Kovačević, and Zora Konjović: Combining CoTraining with Ensemble Learning for Application on Single-View Natural Language Datasets, Acta Polytechnica Hungarica, 10.2, 133-152, 2013

[30] Valenzuela, M., Bentley, J. M., Lorenz, R. D.: Sensorless Tension Control in Paper Machines, Conference Record of the 2002 Annual Pulp and Paper Industry Technical Conference, Vol. 17, No. 21, pp. 44-53, June 2002

[31] Weixuan Liu, Davison, E. J.: Servomechanism Controller Design of Web Handling Systems, IEEE Transactions on Control Systems Technology, Vol. 11, No. 4, pp. 555-564, July 2003 
[32] D. P. D. Whitworth, M. C. Harrison: Tension Variations in Pliable Material in Production Machinery, Applied Mathematical Modelling, Volume 7, Issue 3, pp. 189-196, June 1983

[33] Xiong, T., Cai, W., Xiong, Y. \& Zhang, R.: Dynamic Matrix Control of the Lateral Position of a Moving Web, International Conference on Mechatronics and Automation (ICMA) 1091-1096, 2012

[34] Young G. E., Reid K. N.: Lateral and Longitudinal Dynamic Behavior and Control of Moving Webs, Journal of Dynamic Systems, Measurement, and Control, Vol. 115(2B) pp. 309-317, 1993

[35] http://www.isa-95.com/subpages/technology/isa-95/isa-95.php

[36] http://www.isa-95.com/subpages/advantages/models.php 